\title{
The DEAP-3600 Experiment
}

Mark Stringer ${ }^{a, b, *}$ on behalf of the DEAP-3600 Collaboration

(a complete list of authors can be found at the end of the proceedings)

${ }^{a}$ Department of Physics, Engineering Physics, and Astronomy, Queen's University, Kingston, Ontario, K7L 3N6, Canada

${ }^{b}$ Arthur B. McDonald Canadian Astroparticle Research Institute, Queen's University, Kingston, Ontario, K7L 3N6, Canada

E-mail: m.stringer@owl.phy.queensu.ca

The DEAP-3600 experiment searches for dark matter via the interactions of WIMPs with a liquid argon target. The experiment is located at SNOLAB in Sudbury, Ontario, $2 \mathrm{~km}$ underground to shield the detector from cosmic rays. The detector consists of an acrylic sphere with an inner diameter of $\sim 170 \mathrm{~cm}$ containing $\sim 3300 \mathrm{~kg}$ of liquid argon. Liquid argon is chosen as a target due to its ability to reject electromagnetic backgrounds by examining its scintillation pulse shape. The argon volume is instrumented with 255 PMTs which are connected to the vessel via acrylic light guides. As liquid argon scintillates at a wavelength of $128 \mathrm{~nm}$, its scintillation light needs to be shifted to a wavelength into a region where the PMTs are more sensitive; this is done by coating the inside of the acrylic vessel with TPB wavelength shifter, which re-emits the argon scintillation light at a wavelength of $420 \mathrm{~nm}$. This talk will describe the current status of the experiment and some recent analyses performed by the collaboration. The status of planned upgrades to the detector and the plans for the future of the experiment will also be detailed.

$37^{\text {th }}$ International Cosmic Ray Conference (ICRC 2021)

July 12 th - 23rd, 2021

Online - Berlin, Germany

\footnotetext{
${ }^{*}$ Presenter

$\dagger$ We thank the Natural Sciences and Engineering Research Council of Canada, the Canadian Foundation for Innovation (CFI), the Ontario Ministry of Research and Innovation (MRI), and Alberta Advanced Education and Technology (ASRIP), Queen's University, the University of Alberta, Carleton University, the Canada First Research Excellence Fund, the Arthur B. McDonald Canadian Astroparticle Research Institute, DGAPA-UNAM (PAPIIT No. IN108020) and Consejo Nacional de Ciencia y Tecnología (CONACyT, Mexico, Grant A1-S-8960), the European Research Council Project (ERC StG 279980), the UK Science and Technology Facilities Council (STFC) (ST/K002570/1 and ST/R002908/1), the Leverhulme Trust (ECF-20130496), the Russian Science Foundation (Grant No. 21-72-10065), the Spanish Ministry of Science and Innovation (PID2019-109374GB-I00), and the International Research Agenda Programme AstroCeNT (MAB/2018/7) funded by the Foundation for Polish Science (FNP) from the European Regional Development Fund. Studentship support from the Rutherford Appleton Laboratory Particle Physics Division, STFC and SEPNet PhD is acknowledged. We thank SNOLAB and its staff for support through underground space, logistical, and technical services. SNOLAB operations are supported by the CFI and Province of Ontario MRI, with underground access provided by Vale at the Creighton mine site. We thank Vale for their continuing support, including the work of shipping the acrylic vessel underground. We gratefully acknowledge the support of Compute Canada, Calcul Québec, the Centre for Advanced Computing at Queen's University, and the Computation Centre for Particle and Astrophysics (C2PAP) at the Leibniz Supercomputer Centre (LRZ) for providing the computing resources required to undertake this work.
} 


\section{Introduction}

DEAP-3600 is a WIMP dark matter direct detection experiment [1]. Particle dark matter is well motivated by astronomical observations including galaxy rotation curves [2] and measurements of the Bullet Cluster [3]. The following sections will describe the current status of the experiment and the latest limits set on WIMP dark matter by the experiment. Some of the major backgrounds to the WIMP search are described, and analysis techniques and hardware upgrades to mitigate them are detailed. Other physics analyses which can be performed by the experiment are also described.

\section{The DEAP-3600 Detector}

The DEAP-3600 detector consists of $\sim 3300 \mathrm{~kg}$ of liquid argon (LAr) contained within an acrylic sphere with a radius of $\sim 85 \mathrm{~cm}$. The acrylic sphere is instrumented with 255 high quantum efficiency photomultiplier tubes (PMTs). When the argon is excited by particles it emits light; this light is observed by the PMTs. Liquid argon is chosen as it has a good scintillation light yield and is transparent to its own scintillation light. It is also chosen due to its excellent pulse shape discrimination performance, as described in the next section. TPB wavelength shifter is applied to the inner surface of the acrylic sphere to shift the argon scintillation from $128 \mathrm{~nm}$ to $420 \mathrm{~nm}$ where the PMTs are sensitive. The inner detector is contained within a steel shell which is submerged within a water Cherenkov muon veto tank. An image of the detector can be seen in Figure 1 in [1]. The experiment is located at SNOLAB in Sudbury, Ontario, Canada.

\section{Pulse Shape Discrimination using Liquid Argon}

The use of liquid argon allows powerful separation between electron recoil events (backgrounds) and nuclear recoil events (potential WIMP signal). Argon has two scintillation lifetimes: the singlet state which decays with a lifetime of less than $10 \mathrm{~ns}$, and a triplet state which decays with a lifetime of $\sim 1.43 \mu \mathrm{s}$ [4] [5]. Electron recoil events, which are interactions resulting from $\beta, \gamma$ and muon interactions mainly excite the triplet state of argon and the scintillation light occurs over a period of $\mu \mathrm{s}$. In contrast, when the argon is excited by an $\alpha$, neutron, or WIMP it is mainly excited in the singlet state, resulting in light emission over a period of ns. In DEAP-3600 events are characterized via $\mathrm{F}_{\text {prompt }}$ as described in Eq. 1 [6]. Events where light is emitted promptly will have a high $\mathrm{F}_{\text {prompt }}$. Using pulse shape discrimination is crucial to the success of the experiment. Figure 14 in [6] shows the leakage of ${ }^{39} \mathrm{Ar}$ as a function of $\mathrm{F}_{\text {prompt }}$ : PSD reduces the rate from $3 \mathrm{kHz}$ to less than one event in the entire 231 day dataset, whilst still keeping good WIMP acceptance.

$$
\mathrm{F}_{\text {prompt }}=\frac{\sum_{t=-28 \mathrm{~ns}}^{60 \mathrm{~ns}} \mathrm{PE}(\mathrm{t})}{\sum_{t=-28 \mathrm{~ns}}^{10 \mu \mathrm{s}} \mathrm{PE}(\mathrm{t})}
$$

\section{Degraded And Shadowed Alpha Backgrounds}

Two of the dominant backgrounds to the WIMP dark matter search in the experiment are due to degraded and shadowed alpha decays. 


\subsection{Neck Backgrounds}

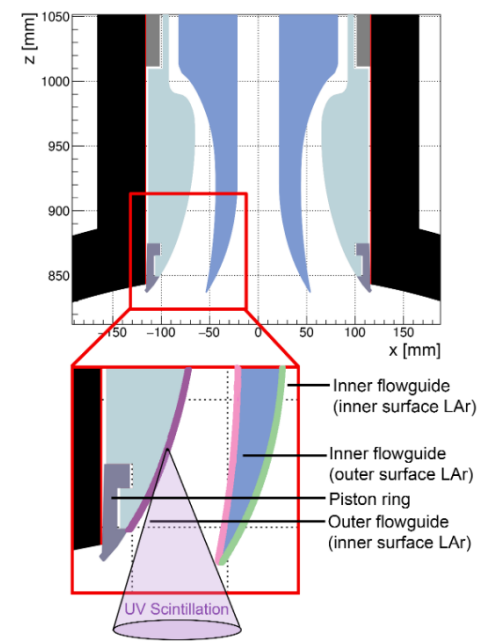

(a) Diagram illustrating the origin of neck alpha backgrounds. [6]

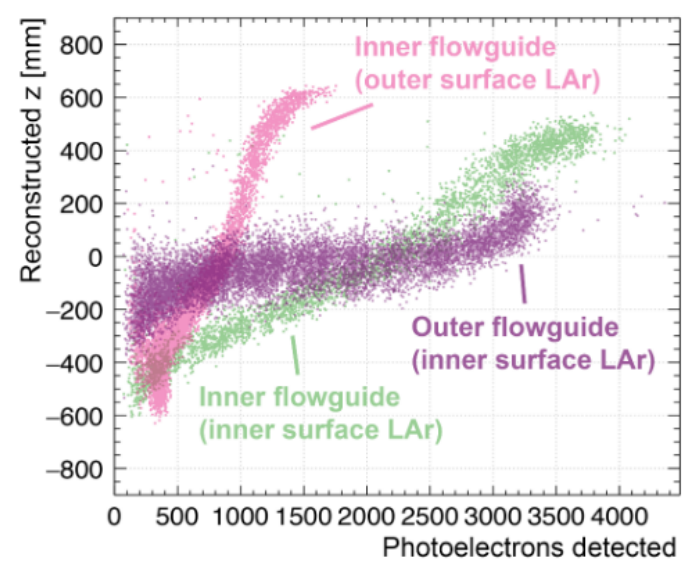

(b) The reconstructed $\mathrm{Z}$ position of events as a function of $\mathrm{PE}$ for simulated neck alpha events. [6]

Figure 1: A schematic of neck events and how they misreconstruct within the detector.

The first of the two backgrounds is due to alpha decays within the neck region of the detector. It is possible that a thin layer of condensed liquid argon coats the flow guides. Another possibility is a mist surrounding the flow guides. This condensation/mist is caused by the cooling setup of the detector. When an alpha decay occurs within the flow guides there is a chance that it can pass through the thin layer of liquid argon or mist producing scintillation light. This scintillation light is then shadowed by the acrylic resulting in the decays having a similar energy to WIMP events. A diagram illustrating the origin of this background is shown in Figure 1a. Furthermore, as the decays are produced by alpha particles they have a high $F_{\text {prompt }}$. At WIMP search energies these decays also reconstruct within the fiducial volume as shown in Figure 1b. For the results in [6] neck alpha events were removed by employing neck veto cuts, fiducial cuts and checking the events reconstruct consistently when using different reconstruction algorithms.

\subsection{Dust Backgrounds}

Another alpha background is due to dust events. A dust event is shown graphically in Figure 2a. These events are thought to be caused by small dust particles within the detector. When an alpha decay occurs within a dust particle some of the alpha's energy is lost in the dust particle as it exits. If the alpha then gets to the liquid argon, the scintillation light is then shadowed by the dust particle. This shadowing and energy loss within the dust can result in these alphas having a similar energy to WIMP NR events. A model of the dust has been developed where the number density of dust particulates with a given radius is based on power law. Simulations of this model show good agreement with data at energies above the WIMP search region as shown in Figure 2b. 

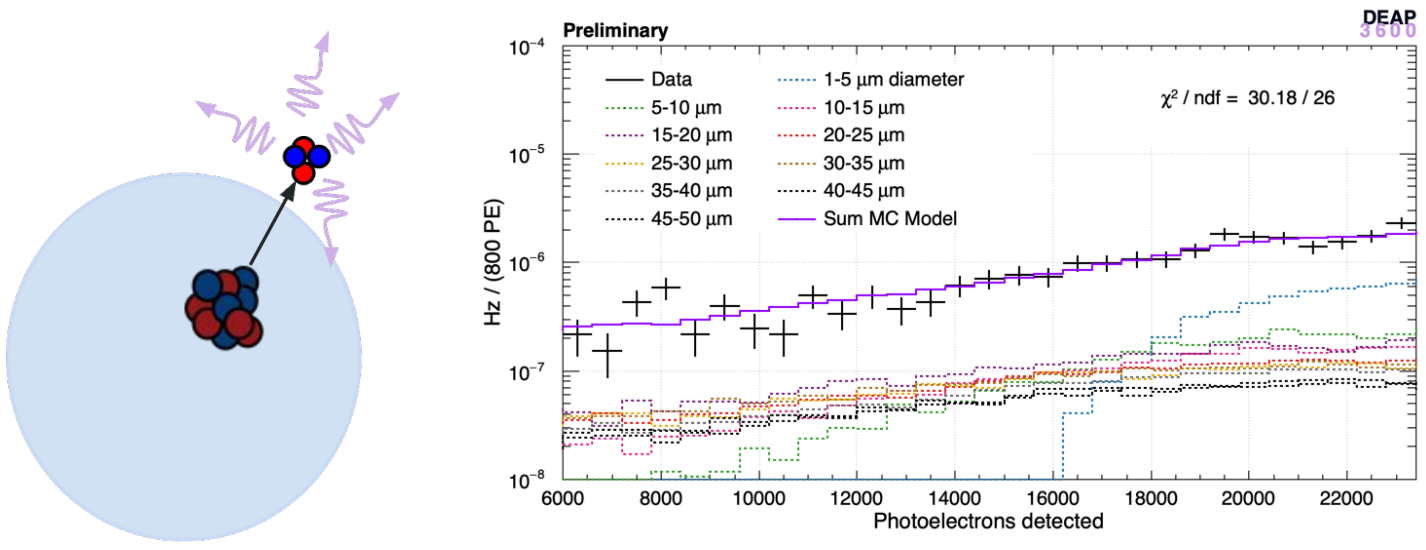

(a) A graphic illustrating a dust (b) The energy distribution of dust events in data compared with a power law model event. used to weight MC simulations of various dust sizes.

Figure 2: A graphic describing the nature of the dust backgrounds and the comparrison between dust simulations and data.

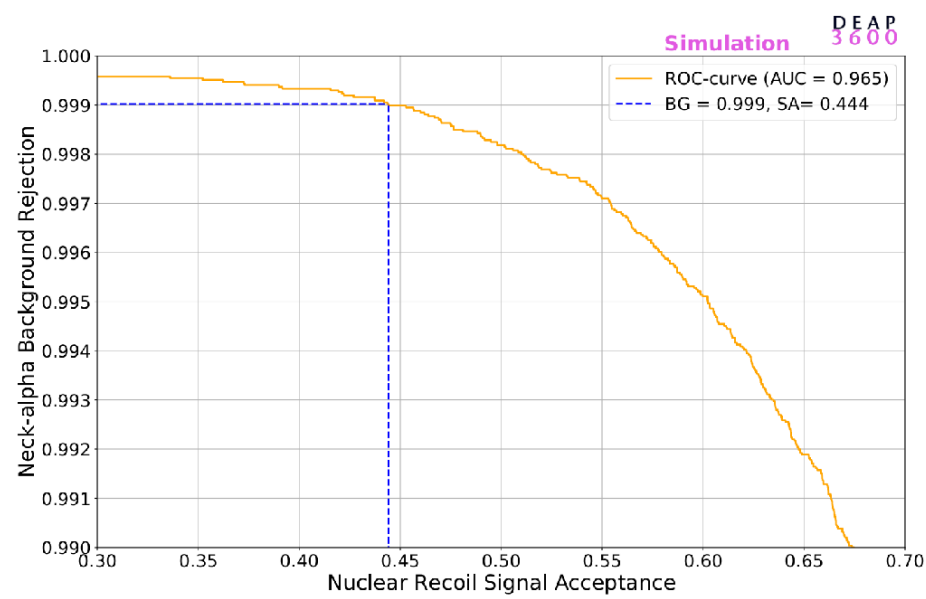

Figure 3: The receiver operating characteristic (ROC) curve for distinguishing neck alpha events from WIMP events using a multilayer perceptron.

\subsection{Multivariate Analysis Techniques}

Work is ongoing to employ multivariate analysis (MVA) techniques to discriminate between neck/dust alpha backgrounds and WIMP signal events. Figure 3 shows the ROC curve for distinguishing neck alpha events and WIMP events using a multilayer perceptron. Neck alpha events are rejected at the $99.9 \%$ level whilst keeping approximately $44 \%$ of the WIMP signal. Work is underway to develop similar MVA techniques to reject dust backgrounds. 


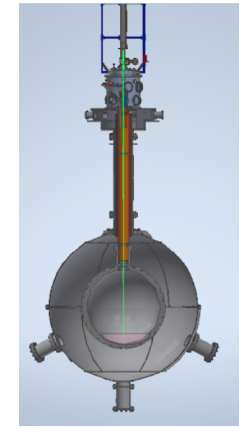

(a) A schematic of the alternative cooling system planned for the upgraded DEAP-3600 experiment.

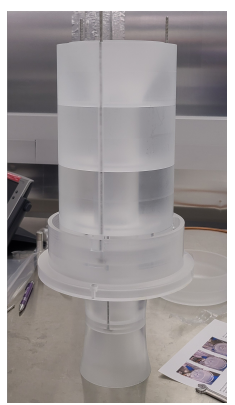

(b) The new neck flow guides for the upgraded DEAP-3600 experiment.

Figure 4: The alternative cooling system and new neck flow guides of the DEAP-3600 detector.

\section{Current Sensitivity Of The Experiment}

The latest results use 231 days of detector livetime. Using a cut and count strategy, backgrounds were constrained such there was less than one expected event in the WIMP search region of interest. No WIMP candidate events were observed.

Figure 24 from [6] shows the current sensitivity of DEAP-3600 in the standard scenario where the standard dark matter halo model is used and the dark matter is assumed to couple equally to neutrons and protons. Alternative galactic halo models and various coupling scenarios are detailed in [7]. Figure 7 in [7] shows the sensitivity of the experiment in the so-called "xenonphobic" regime. In this scenario the dark matter couples differently to the proton and the neutron. This difference results in an improved sensitivity compared to xenon based detectors.

\section{Detector Upgrades}

The installation of upgrades to the DEAP-3600 detector are underway. One of the main objectives of these upgrades is to significantly reduce the degraded alpha backgrounds within the experiment. Furthermore, repairs to the neck seal of the vessel allow the filling of the detector with LAr to its original design capacity.

\subsection{The Alternative Cooling System}

Currently, the LAr within the DEAP-3600 detector is cooled using cooling coils within the neck of the detector. This cooling setup causes one of the backgrounds described in the previous section. The alternative system replaces the original cooling system. In the new system the cooling of the argon is performed external to the detector and piped into the vessel via a tube. A schematic of the alternative cooling system is shown in Figure 4a. As well as cooling the liquid argon the new cooling system allows the filtering of the argon, this is expected to significantly reduce any dust backgrounds within the experiment. 


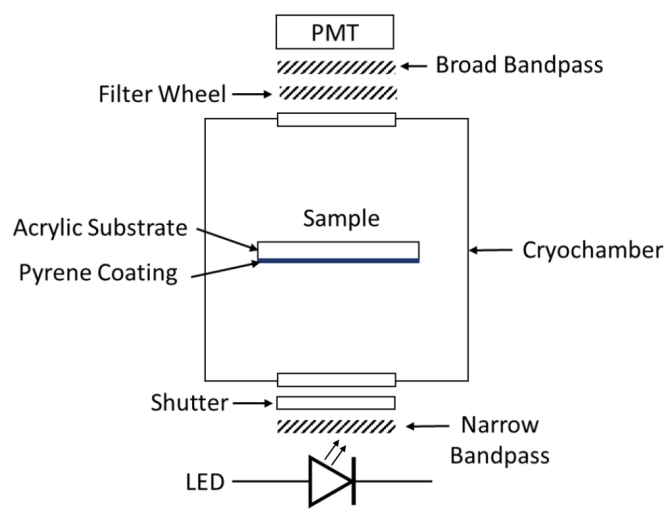

(a) A schematic of the experimental setup at Queen's University used to characterize the flow guide coatings.

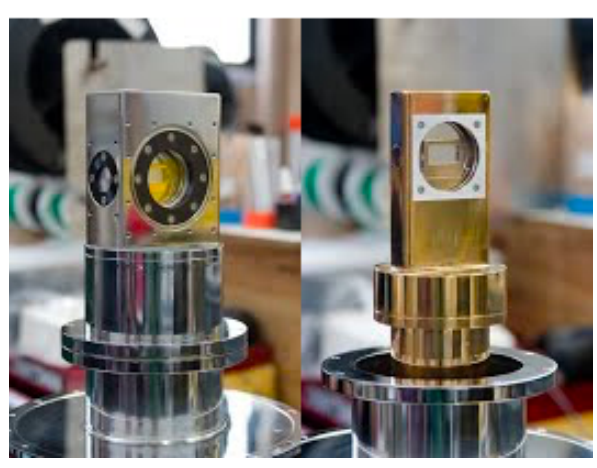

(b) The cryostat with window containing the sample.

Figure 5: The setup at Queen's University used to characterize slow wavelength shifters.

\subsection{Coating The Flow Guides}

During the upgrades the detector flow guides in the neck will be replaced. A picture of the newly machined flow guides is shown in Figure $4 \mathrm{~b}$. Before installation these flow guides will be coated with a pyrene wavelength shifter. The shifter has a significantly longer decay time than the singlet lifetime of argon. After installation any neck alpha events will have a much longer scintillation decay time, and therefore will no longer look like WIMP signal events.

Various wavelength shifters were characterised ex-situ at Queen's University [8]. A schematic of the setup can be seen in Figure 5a. The setup consists of an UV LED which is used to illuminate the sample with a pulse over a period of nanoseconds. A filter is placed between the sample and the LED to ensure the sample is illuminated only with UV light. The sample is viewed via a PMT on the opposite side to the LED. Various filters exist between the sample and the PMT and are used to measure various segments of the emission spectrum. The sample is deposited on an acrylic substrate and contained within a cryostat vessel with quartz windows. This allows the time resolved measurement to be performed at several temperatures between room temperature and $4 \mathrm{~K}$. An image of the cryostat containing the samples can be seen in Figure $5 \mathrm{~b}$.

One of the measurements made using this setup can be seen below in Figure 6 . At $87 \mathrm{~K}$, the operating temperature of DEAP-3600, the sample is seen to emit light over periods of $\mathrm{O}(\mu \mathrm{s})$.

\section{Other Physics Measurements}

\subsection{Multiply interacting Massive Particles}

Multiply interacting massive particles (MIMPs) are a dark matter candidate predicted by several different theories. Unlike WIMPs which scatter rarely within the detector MIMPs have large scattering cross sections and interact readily. In fact the cross section is so large that multiple scatterings occur as the particle passes through the detector. As every MIMP which enters the detector interacts, the size of the detector determines its sensitivity. The large size of DEAP-3600 results in a high sensitivity. The mass range DEAP-3600 is sensitive to ranges from $10^{7} \mathrm{GeV}$ to 


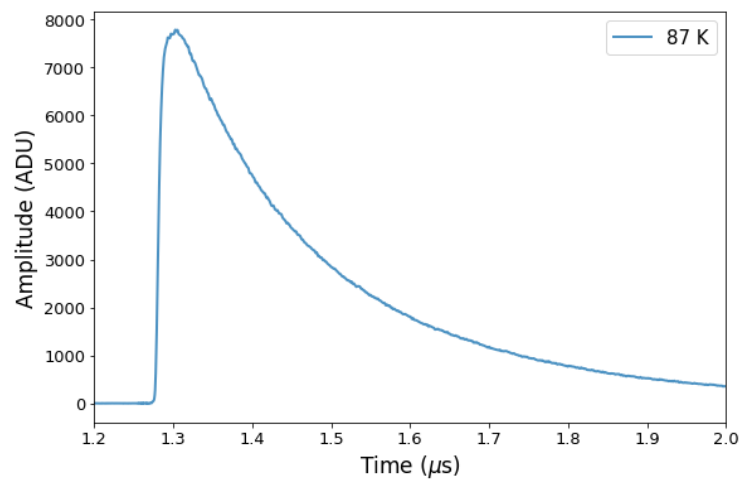

Figure 6: Time resolved fluorescence measurement at $87 \mathrm{~K}$ made using the ex-situ setup at Queen's University of the pyrene wavelength shifter. The pyrene was embedded in a polystyrene matrix and has a concentration of $15 \%$.

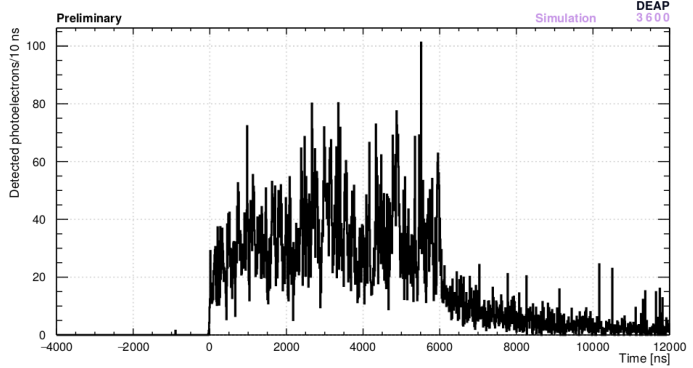

(a) The expected signal due to a MIMP passing through the detector. In this simulated event the interaction cross section is $0.1 \mathrm{mb}$ and the MIMP passes through $85 \mathrm{~cm}$ of liqid argon resulting in approximately 460 scatters.

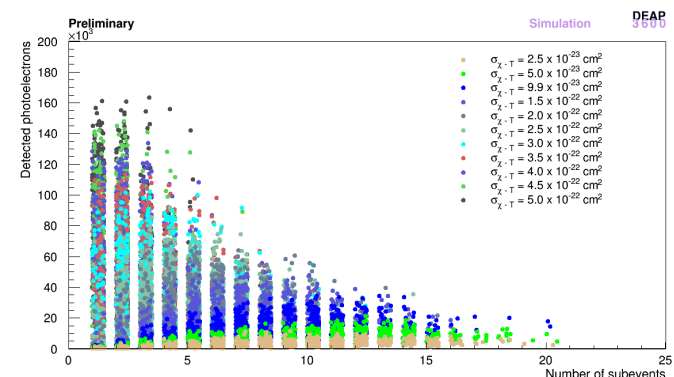

(b) MIMP signal for various cross sections as a function of the number of subevents and the total number of photoelectrons.

Figure 7: The simulated MIMP signal in DEAP-3600.

$10^{19} \mathrm{GeV}$. At these masses, the MIMP is deflected by a very small amount and essentially follows a straight line as it passes through the Earth and the detector.

Simulations have been performed to model the detector response to MIMPs. Figure 7a shows an example of the signal due to a MIMP passing through the detector. Figure $7 \mathrm{~b}$ shows the MIMP signal for various cross sections as a function of the number of subevents (determined by processing the PMT waveform and looking for well separated peaks) and the number of photoelectrons; as the interaction cross section increases the total number of photoelectrons increases due to the increase in the number of scatterings. For higher cross sections the number of subevents decreases, this is due to the fact that the scatterings become so freqent the processing can no longer resolve the individual scatterings. Analysis is underway on the data and results are expected in the near future.

\subsection{Solar Neutrino Measurements}

DEAP-3600 is also sensitive to neutrinos produced in the Sun. The experiment is sensitive to ${ }^{8} \mathrm{~B}$ neutrinos produced via the interaction: ${ }^{8} \mathrm{~B} \rightarrow{ }^{8} \mathrm{Be}+e^{+}+v_{e}(\mathrm{Q}=18 \mathrm{MeV})$. The neutrinos are detected via their absorption on an argon nucleus: $v_{e}+{ }^{40} \mathrm{Ar} \rightarrow{ }^{40} \mathrm{~K}^{*}+e^{-}\left(E_{v_{e}}>3.9 \mathrm{MeV}\right)$ [9], in 
the interaction the electron forms the prompt signal whereas the de-excitation of the ${ }^{40} \mathrm{~K}^{*}$ forms the delayed signal. The golden channel for the delayed signal is the emission of a $1.64 \mathrm{MeV} \gamma$ with a mean decay time of $480 \mathrm{~ns}$ although other decay modes are possible. In total up to $16 v_{e}$ interactions are expected in 3 years of livetime, before event selection and background rejection.

\section{Conclusions}

DEAP-3600 is a single phase liquid argon detector used to search for WIMP dark matter. The experiment has been running since 2016 and dominant backgrounds to the WIMP search have been identified. MVA techniques are being developed to reduce these backgrounds. Furthermore, upgrades are underway on the detector to mitigate these backgrounds. Besides performing WIMP searches the detector can also search for other signals, including other types of dark matter and solar neutrinos.

\section{References}

[1] DEAP-3600 collaboration, Design and Construction of the DEAP-3600 Dark Matter Detector, Astropart. Phys. 108 (2019) 1 [1712 . 01982].

[2] V.C. Rubin, W.K. Ford, Jr. and N. Thonnard, Extended rotation curves of high-luminosity spiral galaxies. IV. Systematic dynamical properties, Sa through Sc, Astrophys. J. Lett. 225 (1978) L107.

[3] D. Clowe, A. Gonzalez and M. Markevitch, Weak lensing mass reconstruction of the interacting cluster 1E0657-558: Direct evidence for the existence of dark matter, Astrophys. J. 604 (2004) 596 [astro-ph/0312273].

[4] E. Morikawa, R. Reininger, P. Gürtler, V. Saile and P. Laporte, Argon, krypton, and xenon excimer luminescence: From the dilute gas to the condensed phase, The Journal of Chemical Physics 91 (1989) 1469 [https: //doi .org/10 . 1063/1.457108].

[5] DEAP-3600 collaboration, The liquid-argon scintillation pulseshape in DEAP-3600, Eur. Phys. J. C 80 (2020) 303 [2001.09855].

[6] DEAP-3600 collaboration, Search for dark matter with a 231-day exposure of liquid argon using DEAP-3600 at SNOLAB, Phys. Rev. D 100 (2019) 022004 [1902. 04048].

[7] DEAP-3600 collaboration, Constraints on dark matter-nucleon effective couplings in the presence of kinematically distinct halo substructures using the DEAP-3600 detector, Phys. Rev. D 102 (2020) 082001 [2005 . 14667].

[8] H. Benmansour, Characterization of pyrene films for background rejection in liquid argon dark matter experiments, Master's thesis, Queen's University, 2021.

[9] R.S. Raghavan, Inverse Beta Decay of ${ }^{40} \mathrm{Ar}$ : A New Approach for Observing MeV Neutrinos From Laboratory and Astrophysical Sources, Phys. Rev. D 34 (1986) 2088. 


\section{Full Authors List: DEAP-3600 Collaboration}

P. Adhikari ${ }^{5}$, R. Ajaj ${ }^{5,25}$, M. Alpízar-Venegas ${ }^{13}$, D. J. Auty ${ }^{1}$, H. Benmansour ${ }^{19}$, C.E. Bina ${ }^{1,25}$, W. Bonivento ${ }^{9}$, M. G. Boulay ${ }^{5}$, M. Cadeddu ${ }^{3,9}$, B. Cai ${ }^{5,25}$, M. Cárdenas-Montes ${ }^{6}$, S. Cavuoti ${ }^{8,7,11}$, Y. Chen ${ }^{1}$, B. T. Cleveland ${ }^{21,12}$, J. M. Corning ${ }^{19}$, S. Daugherty ${ }^{12}$, P. DelGobbo ${ }^{5,25}$, P. Di Stefano ${ }^{19}$, L. Doria ${ }^{18}$, M. Dunford ${ }^{5}$, E. Ellingwood ${ }^{19}$, A. Erlandson ${ }^{5,4}$, S. S. Farahani ${ }^{1}$, N. Fatemighomi ${ }^{21,20}$ G. Fiorillo ${ }^{7,11}$, D. Gallacher ${ }^{5}$, P. García Abia ${ }^{6}$, S. Garg ${ }^{5}$, P. Giampa ${ }^{23}$, D. Goeldi ${ }^{5,25}$, P. Gorel ${ }^{21,12,25}$, K. Graham ${ }^{5}$, A. Grobov ${ }^{15,16}$, A.L. Hallin 1 , M. Hamstra ${ }^{5}$, T. Hugues ${ }^{2}$, A. Ilyasov ${ }^{15,16}$, A. Joy ${ }^{1,25}$, B. Jigmeddorj ${ }^{4}$, C. J. Jillings ${ }^{21,12}$, O. Kamaev ${ }^{4}$, G. Kaur ${ }^{5}$, A. Kemp ${ }^{19,20}$, I. Kochanek ${ }^{10}$, M. Kuźniak ${ }^{2,5,25}$, M. Lai ${ }^{3,9}$, S. Langrock ${ }^{12,25}$, B. Lehnert ${ }^{5, \text { a }}$, A. Leonhardt ${ }^{24}$, N. Levashko ${ }^{15,16}$,

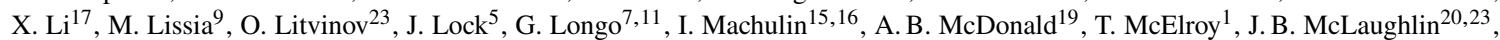
C. Mielnichuk ${ }^{1}$, L. Mirasola ${ }^{3}$, J. Monroe ${ }^{20}$, G. Oliviéro ${ }^{5,25}$, S. Pal ${ }^{1,25}$, S. J. M. Peeters ${ }^{22}$, M. Perry ${ }^{5}$, V. Pesudo ${ }^{6}$, E. Picciau ${ }^{9}, 3$, M.C. Piro ${ }^{1,25}$, T. R. Pollmann ${ }^{24, \text { b }}$, E. T. Rand ${ }^{4}$, C. Rethmeier ${ }^{5}$, F. Retière ${ }^{23}$, I. Rodríguez-García ${ }^{6}$, L. Roszkowski ${ }^{2}{ }^{14}$, J. B. Ruhland ${ }^{24}$, E. Sanchez García ${ }^{6}$, T. Sánchez-Pastor ${ }^{6}$, R. Santorelli ${ }^{6}$, S. Seth ${ }^{5}$, D. Sinclair ${ }^{5}$, P. Skensved ${ }^{19}$, B. Smith ${ }^{23}$, N. J. T. Smith ${ }^{21,12}$, T. Sonley ${ }^{21,25}$, R. Stainforth ${ }^{5}$, M. Stringer ${ }^{19,25}$, B. Sur ${ }^{4}$, E. Vázquez-Jáuregui ${ }^{13,12}$, S. Viel ${ }^{5,25}$, J. Walding ${ }^{20}$, M. Waqar ${ }^{5,25}$, M. Ward $^{19}$, S. Westerdale ${ }^{9,5}$, J. Willis ${ }^{1}$, A. Zuñiga-Reyes ${ }^{13}$,

${ }^{1}$ Department of Physics, University of Alberta, Edmonton, Alberta, T6G 2R3, Canada ${ }^{2}$ AstroCeNT, Nicolaus Copernicus Astronomical Center, Polish Academy of Sciences, Rektorska 4, 00-614 Warsaw, Poland ${ }^{3}$ Physics Department, Università degli Studi di Cagliari, Cagliari 09042, Italy ${ }^{4}$ Canadian Nuclear Laboratories, Chalk River, Ontario, K0J 1J0, Canada ${ }^{5}$ Department of Physics, Carleton University, Ottawa, Ontario, K1S 5B6, Canada ${ }^{6}$ Centro de Investigaciones Energéticas, Medioambientales y Tecnológicas, Madrid 28040, Spain ${ }^{7}$ Physics Department, Università degli Studi "Federico II" di Napoli, Napoli 80126, Italy ${ }^{8}$ Astronomical Observatory of Capodimonte, Salita Moiariello 16, I-80131 Napoli, Italy ${ }^{9}$ INFN Cagliari, Cagliari 09042, Italy ${ }^{10}$ INFN Laboratori Nazionali del Gran Sasso, Assergi (AQ) 67100, Italy ${ }^{11}$ INFN Napoli, Napoli 80126, Italy ${ }^{12}$ Department of Physics and Astronomy, Laurentian University, Sudbury, Ontario, P3E 2C6, Canada ${ }^{13}$ Instituto de Física, Universidad Nacional Autónoma de México, A. P. 20-364, México D. F. 01000, México ${ }^{14} \mathrm{BP} 2$, National Centre for Nuclear Research, ul. Pasteura 7, 02-093 Warsaw, Poland ${ }^{15}$ National Research Centre Kurchatov Institute, Moscow 123182, Russia ${ }^{16}$ National Research Nuclear University MEPhI, Moscow 115409, Russia ${ }^{17}$ Physics Department, Princeton University, Princeton, NJ 08544, USA ${ }^{18}$ PRISMA $^{+}$, Cluster of Excellence and Institut für Kernphysik, Johannes Gutenberg-Universität Mainz, 55128 Mainz, Germany ${ }^{19}$ Department of Physics, Engineering Physics, and Astronomy, Queen's University, Kingston, Ontario, K7L 3N6, Canada ${ }^{20}$ Royal Holloway University London, Egham Hill, Egham, Surrey TW20 0EX, United Kingdom ${ }^{21}$ SNOLAB, Lively, Ontario, P3Y 1N2, Canada ${ }^{22}$ University of Sussex, Sussex House, Brighton, East Sussex BN1 9RH, United Kingdom ${ }^{23}$ TRIUMF, Vancouver, British Columbia, V6T 2A3, Canada ${ }^{24}$ Department of Physics, Technische Universität München, 80333 Munich, Germany ${ }^{25}$ Arthur B. McDonald Canadian Astroparticle Physics Research Institute, Queen's University, Kingston ON K7L 3N6,Canada

${ }^{a}$ Currently: Nuclear Science Division, Lawrence Berkeley National Laboratory, Berkeley, CA 94720

${ }^{\mathrm{b}}$ Currently: Nikhef and the University of Amsterdam, Science Park, 1098XG Amsterdam, Netherlands 